(C) 1980. The Genetical Society of Great Britain

\title{
OSCILLATIONS OF FREQUENCY IN BATESIAN MIMICS, HAWKS AND DOVES, AND OTHER SIMPLE FREQUENCY DEPENDENT POLYMORPHISMS
}

\author{
JOHN R. G. TURNER \\ Department of Genetics, University of Leeds, Leeds LS2 9JT
}

Received 4.i.80

\begin{abstract}
SUMMARY
It is customary to infer the properties of the internal equilibria produced by frequency dependent selection from the properties of the boundary equilibria (often called the "invasion" criterion). This paper demonstrates that there are some circumstances under which a pair of unstable boundary equilibria do permit the conclusion that there is a truly stable, unique, internal equilibrium. For two alleles with complete dominance, if phenotype fitness declines monotonically with increasing frequency, then the internal point of equal phenotype fitness is the unique internal equilibrium of the genes, and is not unstable; this criterion may also be met if the fitness of one phenotype increases with frequency. It must be truly stable, in the sense of not producing oscillations, if the decline of fitness is linear or convex upwards and no phenotype is lethal at any frequency; the hawk-dove game complies with both conditions, and at least the second condition is likely to be met in most of the models encountered in sociobiology. However, an equilibrium which induces damped oscillations, or perhaps even complex limit cycles, is possible if at least one phenotype can be lethal at high frequency, or if the decline in fitness is strongly curvilinear and concave upwards.

One case of curvilinear frequency dependence, a dimorphic batesian mimic with a non-mimetic form, is examined in detail. Although oscillations about the equilibrium point are possible, no matter whether the mimicry is dominant, recessive or $Y$-linked, this will only occur when selection coefficients are very large, and (except for Y-linkage) only if both sexes can be mimetic. As selection is density as well as frequency dependent, such conditions may be produced in the real world by large fluctuations in population size.
\end{abstract}

\section{INTRODUCTION}

SIMPLE frequency dependent systems are of considerable interest in evolutionary biology: for example in various conflict games in the evolution of social behaviour (Maynard Smith and Price, 1973) the fitness of each behavioural phenotype may decline with increasing frequency, giving a stable, non-trivial equilibrium point, called the evolutionarily stable strategy (ESS), where both phenotypes have equal fitness (fig. 1(a)). But such examples are usually investigated without reference to the underlying genotypes, and the conclusions drawn therefore apply strictly only to a haploid organism, or to competing clones or competing non-interbreeding species. To what extent it is legitimate to apply these conclusions to diploid sexual organisms?

This is a large question; the simplest case, that of two alleles with complete dominance can be investigated quite easily for three properties: the position and uniqueness of the apparent equilibrium, and its stability, in particular whether it is truly stable or whether displacements of the population from the equilibrium will result in oscillations. 
This paper will investigate this problem for batesian mimicry, a real and quite well understood example of frequency dependence already investigated in some detail (Turner, 1978, 1979), and then for the general case, which unlike batesian mimicry requires solution by numerical methods. The hawk-dove conflict model is a straightforward example of this general case.

\section{Position AND uniqueness of THE EQuilibrium-General CASE}

Is the equilibrium we find by examining the fitness of two phenotypes the same as the one we find by studying the behaviour of genes in a diploid population? We will assume two alleles, complete dominance, and a system of mating which does not alter gene frequencies.

Suppose that there is a population consisting of two forms, form " 0 " and form " 1 ", with frequencies $P_{0}$ and $P_{1}$, whose fitnesses are frequency dependent monotonically and negatively, so that in a population of pure " 0 ", form " 1 " is the fitter, and conversely in a pure " 1 " population, so that there is some non-trivial frequency $\hat{P}_{0}$ at which the fitnesses, say $W_{0}$ and $W_{1}$, of the two forms are equal (fig. 1(a) - the frequency dependence need not be

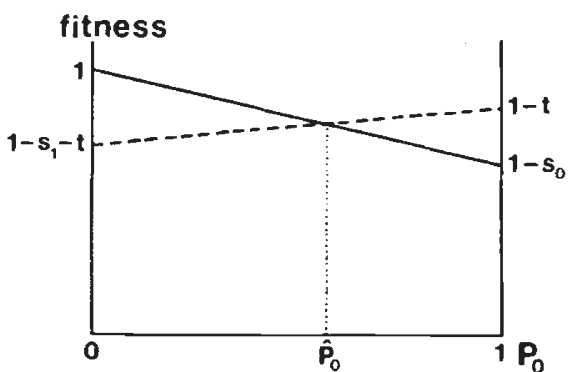

(a)

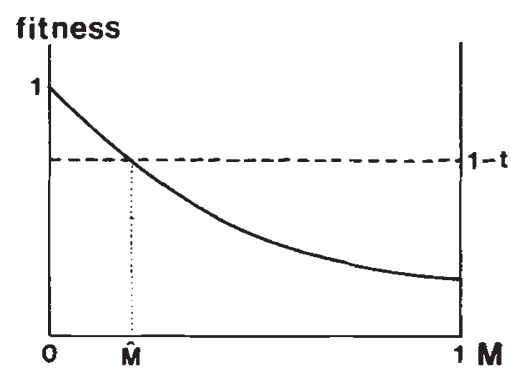

(b)

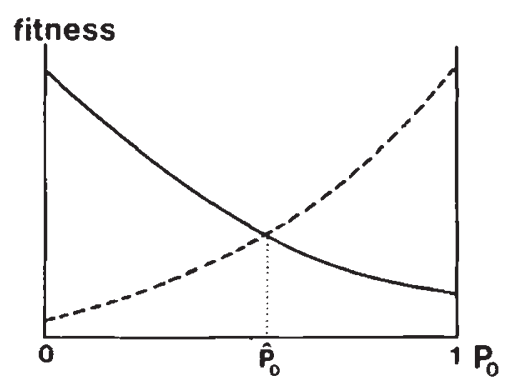

(c)

Fig. 1.-Three theoretical models of frequency dependence, plotted as fitness of phenotype " 0 " (solid line) and of phenotype " 1 " (broken line) against frequency of phenotype " 0 " $\left(P_{0}\right)$. Equilibrium frequency projected by dotted line. (a) General linear case, (b) Polymorphic batesian mimicry, slightly simplified by having the fitness of the non-mimic (broken line) frequency independent, (c) Monotonic, curvilinear case, giving oscillations with no lethality.

linear, but must be monotonic, otherwise there may be three or more equilibria; everything that follows applies to the slightly more general case in which the fitness of only one of the forms increases with its frequency, provided that of the other form is negatively frequency dependent, so that 
there is still a single point of equal fitness). In a haploid, clonal or two species system $\hat{P}_{0}$ is clearly an equilibrium, and the behaviour of mutants or invaders introduced into the pure populations leads us to believe it is stable.

Is this point also the uniquely non-trivial equilibrium in a diploid system of two alleles, in which the two phenotypes are constituted of three genotypes? Suppose, without loss of generality, that form " 0 " is dominant. Under any system of mating which does not in itself change the gene frequency, and this includes random mating, an equilibrium of gene frequency will occur only if there is no change of gene frequency within a generation between zygotes and adults. Thus if the fitness of the two phenotypes are $W_{0}, W_{1}$, the frequencies of the three genotypes $Q_{00}, 2 Q_{01}$, $Q_{11}$, and of the two alleles $q_{0}, q_{1}$, then in the zygotes

$$
q_{0}=Q_{00}+Q_{01}, \quad q_{1}=Q_{01}+Q_{11}
$$

and in the adults

$$
q_{0}^{\prime}=W_{0}\left(Q_{00}+Q_{01}\right) / \bar{w}, \quad q_{1}^{\prime}=\left(W_{0} Q_{01}+W_{1} Q_{11}\right) / \bar{w}
$$

the prime denoting the adults and $\bar{w}$ being the arithmetic mean of fitness of all three genotypes:

$$
\bar{w}=W_{0} Q_{00}+2 W_{0} Q_{01}+W_{1} Q_{11} \text {. }
$$

At equilibrium, by definition $q_{0}^{\prime}=q_{0}$ and $q_{1}^{\prime}=q_{1}$, so that $q_{0}^{\prime} / q_{1}^{\prime}=q_{0} / q_{1}$. Hence

$$
\frac{W_{0}\left(Q_{00}+Q_{01}\right)}{W_{0} Q_{01}+W_{1} Q_{11}}=\frac{Q_{00}+Q_{01}}{Q_{01}+Q_{11}}
$$

or

$$
W_{0}\left(Q_{01}+Q_{11}\right)=W_{0} Q_{01}+W_{1} Q_{11}
$$

which reduces to

$$
W_{0}=W_{1} .
$$

But this is the point of equal phenotype fitnesses. This is therefore itself an equilibrium of gene frequency, and there is no other non-trivial equilibrium. Slatkin (1979) has recently given a more general version of this theorem. The conclusion clearly would not necessarily apply if the genotypes had further effects such that there was some constant difference in fitness between the heterozygote and the dominant homozygote, or if gene frequencies changed at mating.

\section{Special CASE-BATESIAN MIMIC AND NON-MIMIC}

The fitness of a batesian mimic declines as its numbers increase, probably in an asymptotic curve (fig. 1(b)), whose descending limb can without great loss of accuracy be approximated by a straight line. It often happens that a butterfly is polymorphic for a mimetic form and a non-mimetic form: as it leads into the general case, we will assume initially that both sexes are polymorphic. The non-mimic's fitness will be assumed to be frequency independent, with a value of $1-t$. The mimic has a fitness of 1 when rare, 
declining by a fraction $S$ for each mimetic individual added to the population. Let $s_{0}=S N$, where $N$ is the total population size, and $M$ be the frequency of the mimetic phenotype. In terms of the general case above, $M=P_{0}$. Then consideration of the graph (fig. 1(b)) shows a stable equilibrium at the intersection of the two lines, when the fitnesses of both forms are equal, that is when

$$
1-s_{0} M=1-t
$$

showing that the equilibrium frequency of the mimic is (Turner, 1978)

$$
\hat{M}=t / s_{0}=t / S N \text {. }
$$

As it follows that $N \hat{M}=t / S$, this equilibrium has the rather unusual property (Charlesworth and Charlesworth, 1976) that the number of mimics at equilibrium is a constant independent of $N$, the total population size.

We have already shown that this equilibrium is also an equilibrium for an underlying system of two alleles at a single locus, and that there are no other non-trivial equilibria in such a system.

To investigate the stability of the equilibrium, let the fitnesses of the three genotypes be $w_{00}, w_{01}, w_{11}$, so that the change in gene frequency per generation is

$$
\Delta q_{0}=q_{0} q_{1}\left(w_{0}-w_{1}\right) / \bar{w}
$$

where

$$
\begin{aligned}
& w_{0}=q_{0} w_{00}+q_{1} w_{01} \\
& w_{1}=q_{0} w_{01}+q_{1} w_{11} \\
& \bar{w}=q_{0} w_{0}+q_{1} w_{1} .
\end{aligned}
$$

The stability of the equilibrium at $w_{0}-w_{1}=0$ is tested by the value of

$$
\left.\frac{d\left(\Delta q_{0}\right)}{d q_{0}}\right|_{\hat{q}_{0}}=\left.\frac{\hat{q}_{0} \hat{q}_{1}}{\bar{w}}\left\{\frac{d\left(w_{0}-w_{1}\right)}{d q_{0}}\right\}\right|_{\hat{q}_{0}}
$$

where $\hat{q}_{0}$ is the equilibrium gene frequency (e.g., Cook, 1971).

Three genetically different cases are to be investigated, all of which, with the further complication of sex-limitation, are known in mimetic butterflies: haploid (or Y-linked), diploid with the mimic dominant, and diploid with the mimic recessive (respectively Papilio glaucus, most mimics investigated, and Ethiopian Papilio dardanus). The fitnesses are:

$\begin{array}{lccc}\text { Fitness } & \text { Haploid } & \text { Dominant mimic } & \text { Recessive mimic } \\ w_{0} \text { or } w_{00} & 1-s_{0} q_{0} & 1-s_{0}\left(1-q_{1}^{2}\right) & 1-s_{0} q_{0}^{2} \\ w_{01} & - & 1-s_{0}\left(1-q_{1}^{2}\right) & 1-t \\ w_{1} \text { or } w_{11} & 1-t & 1-t & 1-t\end{array}$

(the $w_{i}$ applying to the haploid case, the $w_{i j}$ to the diploid). In all cases $\bar{w}$ at equilibrium is $1-t$, and the equilibrium mimetic phenotype frequency is $t / s_{0}$. The equilibrium gene frequencies are (Turner, 1978): 


$$
\begin{array}{cccc} 
& \text { Haploid } & \text { Dominant mimic } & \text { Recessive mimic } \\
\hat{q}_{0} & t / s_{0} & 1-\sqrt{ }\left\{\left(s_{0}-t\right) / s_{0}\right\} & \sqrt{ }\left(t / s_{0}\right) \\
\hat{q}_{1} & 1-t / s_{0} & \sqrt{ }\left\{\left(s_{0}-t\right) / s_{0}\right\} & 1-\sqrt{ }\left(t / s_{0}\right) .
\end{array}
$$

Then the conditions for the equilibria to be stable are (from 11):

Haploid

$$
0>-\hat{q}_{0} \hat{q}_{1} s_{0} /(1-t)>-1
$$

Dominant mimic

$$
0>-2 \hat{q}_{0} \hat{q}_{1}\left(s_{0}-t\right) /(1-t)>-1
$$

Recessive mimic

$$
0>-2 \hat{q}_{0} \hat{q}_{1} t /(1-t)>-1
$$

where the values of $\hat{q}_{0}$ and $\hat{q}_{1}$ must be obtained from (13). If the left hand inequality is not satisfied, the equilibrium is unstable. Clearly none of the cases is unstable (in (14b), $s_{0}$ must exceed $t$ for the equilibrium to exist). If the right hand inequality is not satisfied then the population will undergo damped oscillations about the equilibrium point (or oscillations of increasing amplitude when the value is less than -2) (Lewontin, 1958 for the general method).

Rearrangement of (14) shows that the values of $t$ which must be exceeded for the equilibria to oscillate are:

Haploid

$$
t>1 /\left(2-\hat{q}_{0}\right) \text { or } \quad t>1 /(2-\hat{M})
$$

Dominant mimic

$$
t>1 /\left[1+2 \hat{q}_{0} \hat{q}_{1}\left\{\left(1-\hat{q}_{1}^{2}\right)^{-1}-1\right\}\right] \text { or } t>1 /\{1+2 \hat{A}(\sqrt{\hat{A}}-\hat{A}) / \hat{M}\}
$$

Recessive mimic

$$
t>1 /\left(1+2 \hat{q}_{0} \hat{q}_{1}\right) \quad \text { or } \quad t>1 /\{1+2(\sqrt{ } \hat{M}-\hat{M})\}
$$

where $A=1-M$ is the frequency of the non-mimetic (usually ancestral) form, and $\hat{q}_{0}, \hat{q}_{1}$ are the values given in (13). In all three cases, the value of $s_{0}$ needed to produce the given value of $\hat{M}$ is $s_{0}=t / \hat{M}$.

Figure 2 shows the physical meaning of these inequalities: for any given value of $\hat{M}$, the equilibrium frequency of the mimetic form, the graph shows the greatest fitness $(1-t)$ of the non-mimetic form which will allow an oscillating equilibrium to occur. Fitnesses below the line of the graph give oscillating equilibria; for fitnesses greater than this, the equilibrium is stable. The value of $s_{0}$, which is of course fixed once $\hat{M}$ and $t$ have been chosen, for the critical value of $t$, is shown in the upper graph.

No batesian mimic will undergo oscillation about its equilibrium point unless the mimic when rare is over twice as fit as the non-mimetic form, and if the mimic is recessive then it must be over three times as fit as the non-mimic. The haploid (Y-linked) case and the dominant mimic behave in much the same way, except that for most equilibrium frequencies the haploid is slightly more permissive of oscillations than the dominant. In both of them the fitness of the non-mimic relative to the mimic when rare declines rapidly with increasing equilibrium frequencies, until for very high 

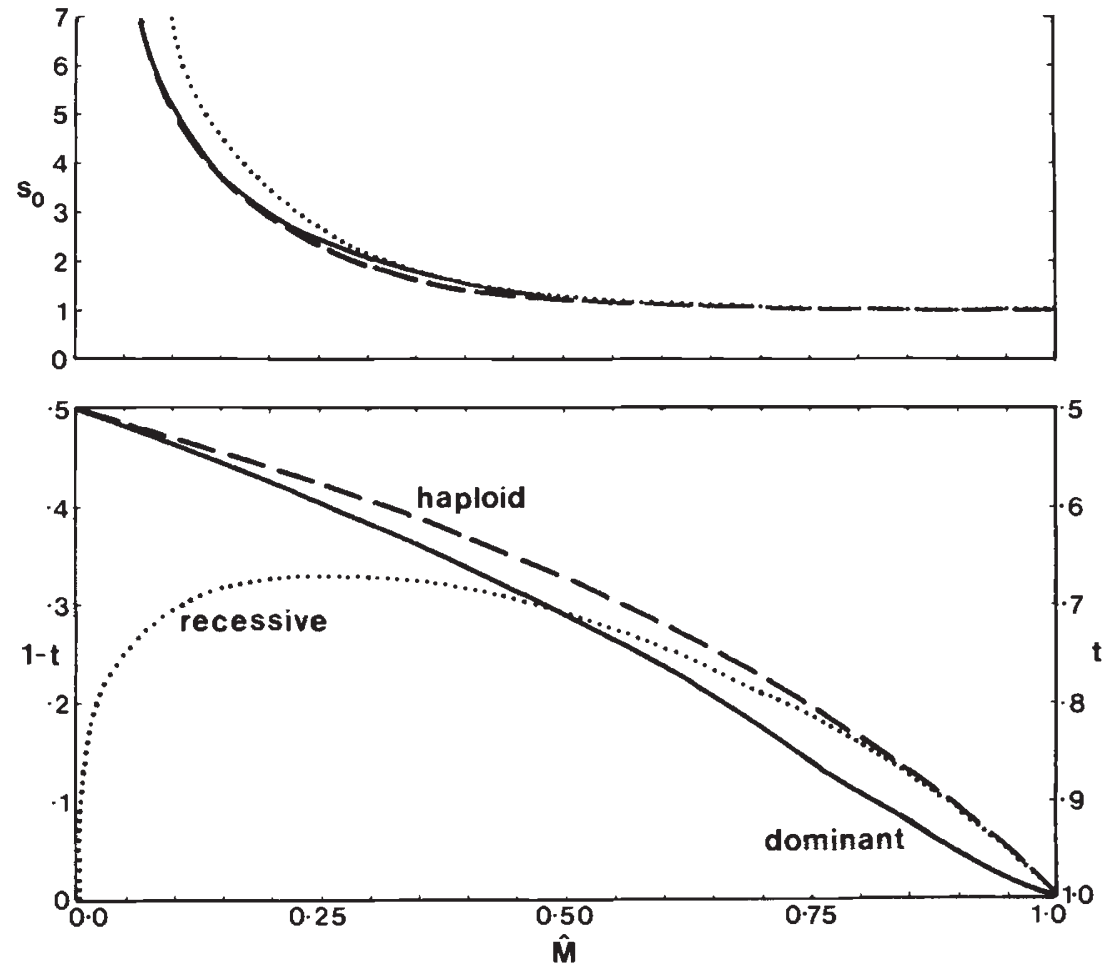

FIG. 2.-Batesian mimicry: critical values of selection parameters required to produce damped oscillations about the equilibrium point, when the equilibrium is at frequency $\hat{M}$. Haploid (Y-linked)-broken line; dominant mimic-solid line; recessive mimic-dotted line. For the diploids, the polymorphism occurs in both sexes.

frequencies, and particularly for the dominant mimic, the non-mimetic form has to be nearly lethal; the same is true for the recessive mimic at high equilibrium frequencies. When it is remembered that for the equilibrium to exist, the mimic when common must be less fit than the non-mimic, it will be seen that it is almost impossible to have an oscillating equilibrium at high frequency. At low frequencies (say below $\hat{M}=0.5$ ) the dominant and haploid more readily permit oscillations (the mimic must be between two and three times as fit as the non-mimic), but then for the equilibrium to exist at this frequency the fitness of the mimic must decline rather rapidly with increasing frequency of the mimic in the population (as shown by the high value of $s_{0}$ ). The recessive mimic is more stringent: if the mimic is as much as four times (but not less than three times) as fit as the mimic when rare, then oscillations are permitted below equilibrium frequencies of around 0.6 and above frequencies of $0 \cdot 05$, the required fitness of the non-mimic declining very rapidly below this frequency (in contrast to the dominant and the haploid).

The conditions needed to produce oscillation are therefore low fitness of the non-mimic compared with the mimic when this is rare, an equilibrium frequency below around 0.6 (not a mathematical necessity, but real cases above this frequency are extremely unlikely), and a mimic whose fitness 
declines rapidly as it becomes more common; with a recessive mimic the decline should not be so rapid as to bring the equilibrium frequency of the mimetic phenotype below around $0 \cdot 05$.

It may seem that these conditions are unlikely to be fulfilled: the mimic has to be very accurate and well protected to be so much fitter than the non-mimic, and at the same time the predators have to perceive that it is different from the model and start predating it once it becomes common, so as to produce a rapid decline in its fitness. However, we have so far treated the system as dependent only on frequency, when it is in fact dependent also on population size. Suppose that in a mimetic species with 1000 individuals, $t=0.6$ (non-mimic 40 per cent as fit as mimic when rare), and $s_{0}=1$, so that the mimetic phenotype has an equilibrium frequency of $0 \cdot 6$. There will be 600 mimics in an equilibrium population, no matter how big the population is, so should the population fluctuate up to 4000 , the equilibrium frequency of the mimics will be only $0 \cdot 15$. As can be seen from fig. 2 , if the mimic is $\mathrm{Y}$-linked or dominant, the equilibrium is now oscillatory, and the oscillations will in fact take place, as the increase in population size will carry the frequency of mimics well above the equilibrium point, and as this is restored by natural selection, the population will approach the equilibrium by a damped oscillation. (A formally equivalent way of looking at this is to note that $s_{0}=S N$, so that $S$ is $0 \cdot 001$, independent of population size. Then in the population of $4000, s_{0}=4, t=0 \cdot 6$.)

Thus dominant and Y-linked mimics are particularly likely to be pushed into an oscillation by an increase in population size; recessive mimics are more complicated, and will become stable again in very large populations.

It is a simple matter to calculate the values of $t$ required for increasing oscillations; they are of course much greater and unlikely to be encountered in real life: for the dominant and Y-linked mimics $t$ must be at least 0.67 (rare mimic three times as fit as non-mimic) at $\hat{M}=0$, and for the recessive $t$ must be at least 0.8 (rare mimic five times as fit as non-mimic) at $\hat{M}=0 \cdot 25$; the curves are the same shape as in fig. 2 . Just how the population behaves with such an equilibrium is an amusing but academic question, until it can be demonstrated that such strong selection is likely to occur; some kind of stable limit cycle seems to be the probable outcome.

However, such oscillations cannot usually occur in the real world because all known mimic-non-mimic polymorphisms in butterflies are confined to the female, the males being non-mimetic. This renders the sex-limited autosomal genes that control the patterns effectively neutral in half the population, and although the equilibrium frequencies remain the same, all selection coefficients are halved (the static gene frequency in the males damping all changes in the females to half speed) (e.g., Charlesworth and Hartl, 1978). As no oscillations can occur in the general case unless $t>0.5$, or more, this means that with sex-limitation, oscillations can never occur, as the non-mimetic form would have to be lethal. (Formally, the numerators in (15b) and (15c) are 2 , and the limiting condition is $t>1$.) Oscillations are possible only with mimic-non-mimic systems that are not sex-limited (a condition which may or may not have been an intermediate step in the evolution of the existing sex-limited polymorphisms). With the Y-linked (haploid) system of Papilio glaucus where the gene does not occur in males, there is no damping effect from that quarter and no alteration is required in the inequalities. The same is true in polymorphisms with both 
forms mimetic, which are frequently found in both sexes, and in which the conditions for oscillation are less stringent, as we will now show.

\section{General case-oscillations}

The above special case has been examined in detail both because it describes a known real polymorphism (a system of mimic and non-mimic), and because it can be explicitly solved. To what extent do the conclusions apply to the general case in which both forms are frequency dependent, which is likely to be found in the hawk-dove and similar games? Confining ourselves to cases where fitness declines monotonically with increasing frequency, consider the model in figure 1(a). The fitnesses of the two forms and the genotypic fitnesses are:

\begin{tabular}{|c|c|c|c|c|c|c|}
\hline \multirow[b]{2}{*}{ Phenotypic fitnesses } & \multicolumn{6}{|c|}{ Genotypic fitnesses } \\
\hline & & Haploid & & 0 "dominan & & " 0 " recessive \\
\hline$W_{0}=1-s_{0} P_{0}$ & $=$ & $w_{0}$ & or & $w_{00}, w_{01}$ & or & $w_{00}$ \\
\hline$W_{1}=1-s_{1} P_{1}-t$ & $=$ & $w_{1}$ & or & $w_{11}$ & or & $w_{01}, w_{11}$ \\
\hline
\end{tabular}

$s_{0}$ and $s_{1}$ being the gradients of the frequency dependent fitness graphs. If we define the following quantities:

$$
\begin{aligned}
& a=s_{1}+t \\
& b=s_{0}-t \\
& c=s_{0}+s_{1} \\
& d=c-s_{0} a
\end{aligned}
$$

then in all three genetic systems the unique equilibrium is

$$
\hat{P}_{0}=a / c, \quad \hat{P}_{1}=b / c
$$

and mean fitness at equilibrium is

$$
\hat{\bar{w}}=1-\left(s_{0} a / c\right) \text {. }
$$

It follows that

$$
s_{0}=\left(a / \hat{P}_{0}\right)-s_{1} .
$$

Substituting (16) in (11) the equilibrium will be stable if:

Haploid

$$
0>-a b / d>-1
$$

"0" dominant

$$
0>-2 b c\{\sqrt{ }(b / c)-(b / c)\} / d>-1
$$

“0” recessive

$$
0>-2 a c\{\sqrt{ }(a / c)-(a / c)\} / d>-1 .
$$

The conditions required to provide non-negative solutions of (18) (existence of non-trivial equilibrium) and to satisfy the left hand inequality (stability in the broad sense) are obvious and not particularly interesting.

Oscillation is produced if the right hand inequality is not satisfied. Numerical solutions of this condition have been obtained by (i) fixing a 
chosen value of $s_{1}$, (ii) fixing a chosen $\hat{P}_{0}$, (iii) choosing a trial value of $t$, (iv) calculating the expression in (21), (v) iterating for $t$ until (21) was equal to -1 . The critical values so computed of $t$ and $s_{0}$ for the complete range of equilibrium phenotype frequencies $\left(\hat{P}_{0}\right)$, for values of $s_{1}$ from -0.25 to 1 are shown in figs. 3-5. The curves are of the same type as in the special case $\left(s_{1}=0\right)$ (fig. 2), which reappears as the next to lowermost curve in these graphs. As expected, now that form " 1 " is allowed some frequency dependence, the fitness of form " 1 " when rare which permits an oscillatory equilibrium can be higher than in the special case. But the curves all have the following features in common: (i) as the equilibrium frequency of form " 0 " approaches 1 , the fitness of form " 1 " when rare, and of form " 0 " when common approach zero; (ii) the fitness of form " 1 " when common (approaching 1) must be no more than half the fitness of form " 0 " when rare for an oscillatory equilibrium to occur, the limit of 50 per cent being approached for an equilibrium frequency $\hat{P}_{0}=0$ (that is to say $s_{1}+t \geqq \frac{1}{2}$; (iii) the critical values of $t$ for the dominant and haploid increase monotonically with decreasing $\hat{P}_{0}$, while for the recessive this reaches a maximum with low $\hat{P}_{0}$ and then decreases to a limiting value of $s_{1}+t=0$ as $\hat{P}_{1}$ approaches 0 (form " 1 " lethal when common); (iv) the diploid cases are less permissive of

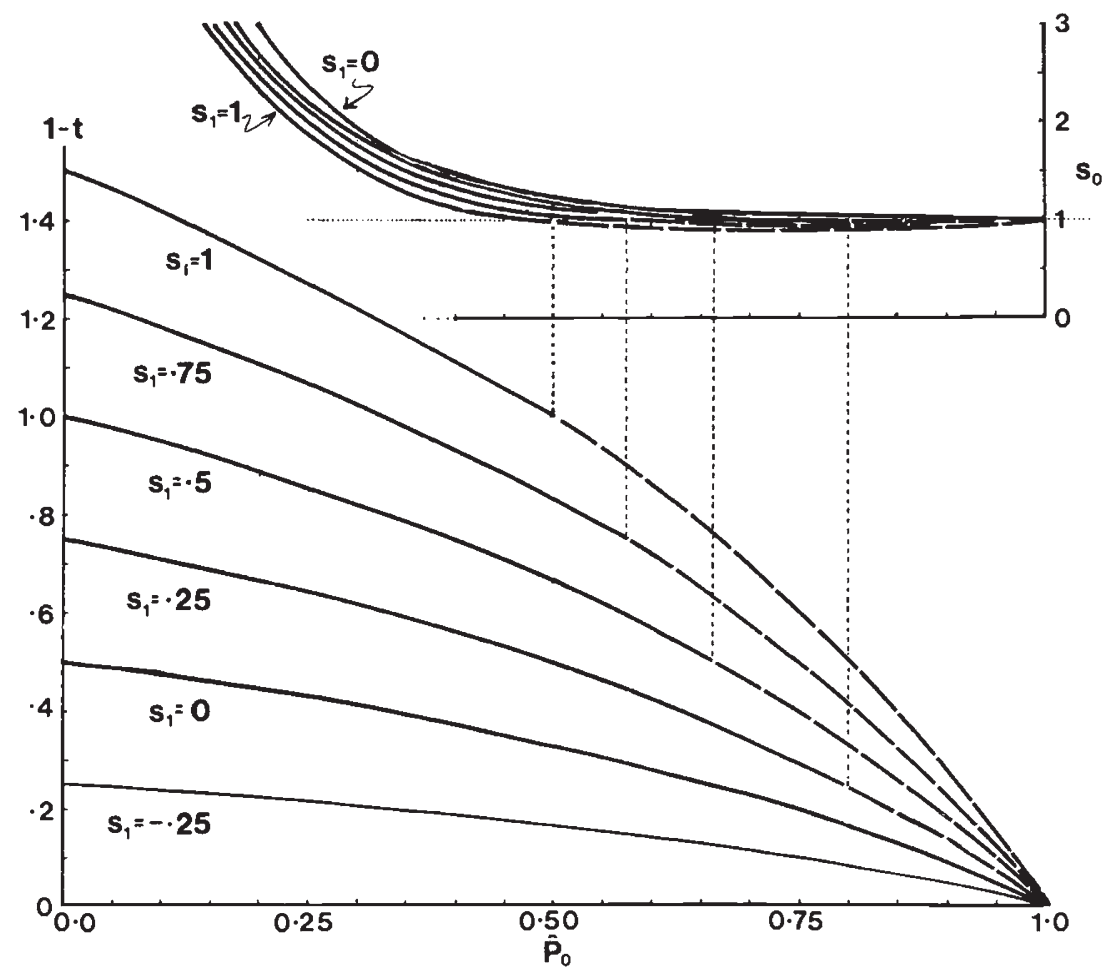

FIG. 3.-General linear case: haploid. Critical values of selection parameters required to produce damped oscillations about the equilibrium at frequency $\hat{P}_{0}$. Solid line indicates that form " 0 " is lethal at some frequencies $\left(s_{0}>1\right)$, dashed line that form " 1 " is lethal at some frequencies $\left(s_{1}+t>1\right)$. Dotted lines connecting graphs show that the domains $s_{0}>1$ and $s_{1}+t>1$ are non-overlapping. In the upper graph the curves for $s_{1}$ negative lie just above that for $s_{1}=0$. 


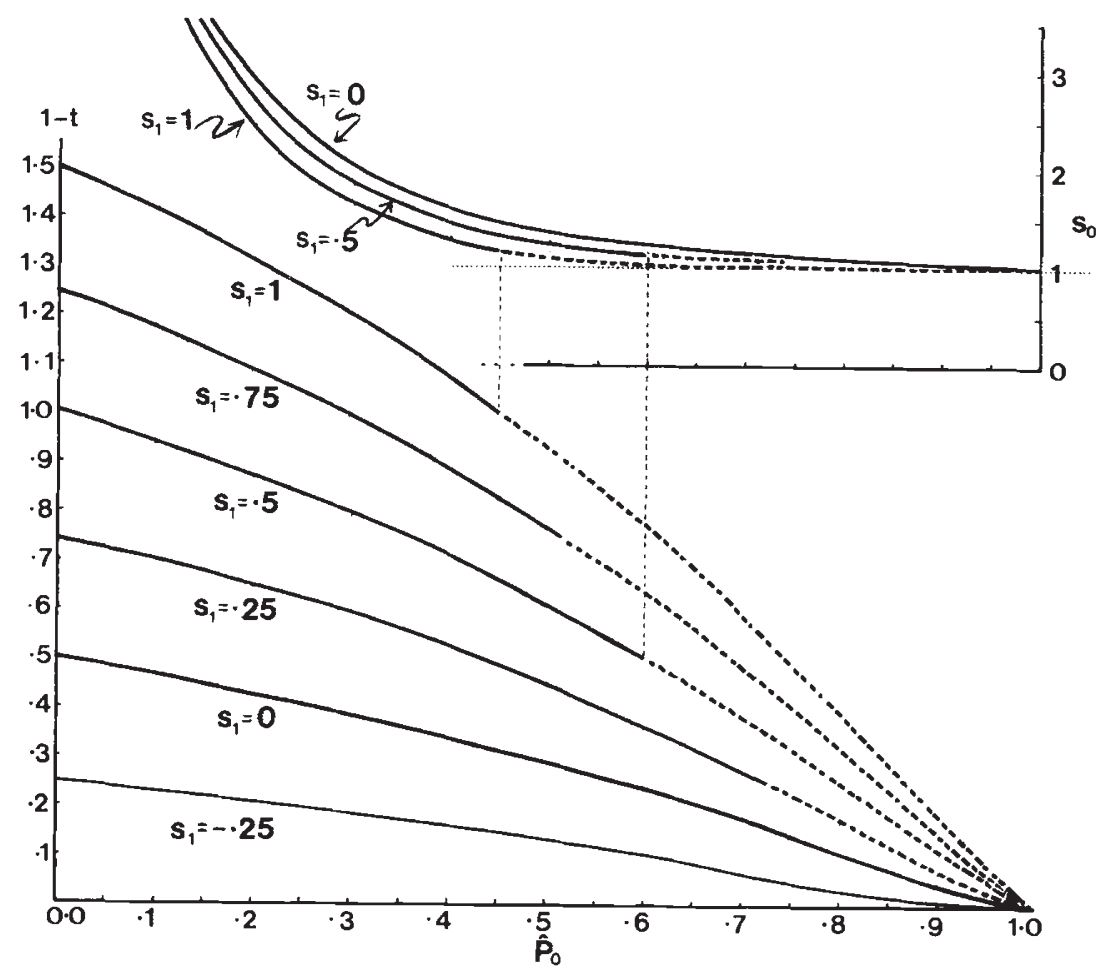

FIG. 4.-General linear case, form " 0 " dominant. As fig. 3. Solid line indicates that form " 0 " is lethal at some frequencies $\left(s_{0}>1\right)$; finely dashed line indicates that both form " 0 " and form " 1 " are lethal at some frequencies $\left(s_{0}>1\right.$ and $\left.s_{1}+t>1\right)$.

oscillations than the haploid, the critical value of $t$ for form " 0 " dominant approaching the haploid asymptotically when $\hat{P}_{0}$ is low, and that for the recessive approaching the haploid asymptotically when $\hat{P}_{0}$ is high; $(\mathrm{v})$ the critical values of $t$ for the dominant and recessive are equal when $\hat{P}_{0}=0.5 ;$ (vi) in all cases the critical value of $s_{0}$ (the gradient of frequency dependence of form " 0 ") is around 1 (or $45^{\circ}$ downward) for values of $\hat{P}_{0}$ above $0 \cdot 5$, being greater or less than 1 according to the case, but increases rapidly up to infinity as $\hat{P}_{0}$ tends to zero; (vii) for negative values of $s_{1}$, (fitness of " 1 " positively frequency dependent), the conditions are extremely stringent and $s_{0}>1$ for all equilibrium frequencies.

But the important finding is that for all equilibrium phenotype frequencies, oscillations are permitted only with selection coefficients that are so large that one or other of the forms is lethal when or before its frequency reaches 100 per cent of the population; that is either $s_{0}>1$ or $s_{1}+t>1$ (in figs. 3-5 solid lines indicate that form " 0 " is lethal, dashed lines that form " 1 " is lethal under these circumstances); for the recessive, there is a narrow range of equilibrium frequency where both forms must be lethal when common for the equilibrium to be oscillatory, and this is true for a wide range of frequency in the dominant case (dotted line in figs. 3-5). Thus if the biological nature of the problem precludes lethality of both forms (and this would certainly be the case with most sensible versions of the animal conflict game), then oscillation around the equilibrium point is also excluded. 


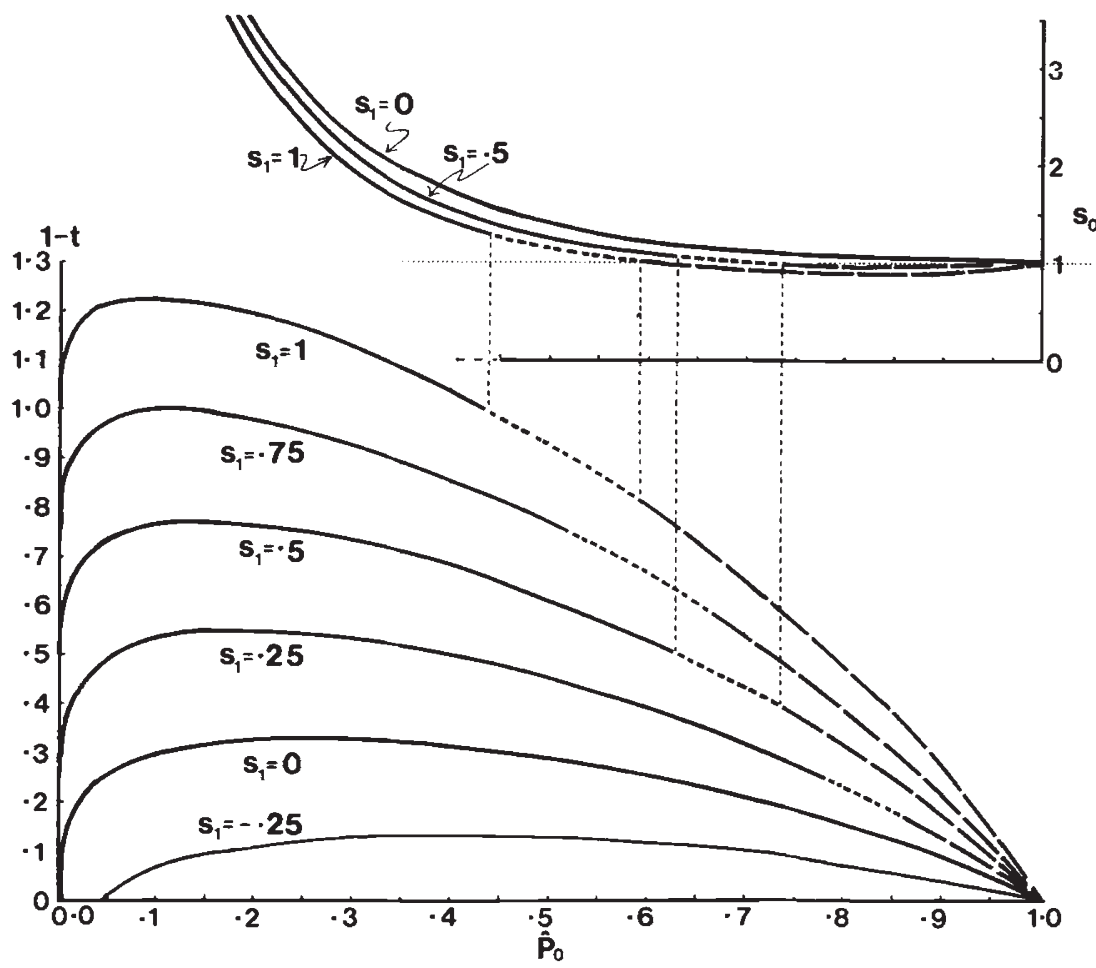

FIG. 5.-General linear case, form " 0 " recessive. As fig. 3. Solid line indicates that form " 0 " is lethal at some frequencies $\left(s_{0}>1\right)$, dashed line that form " 1 " is lethal at some frequencies $\left(s_{1}+t>1\right)$. The overlap of these domains, where both forms are lethal at some frequencies $\left(s_{0}>1\right.$ and $\left.s_{1}+t>1\right)$ is shown by the finely dashed line.

However, if the graphs of frequency dependence of the phenotypes are not linear, as is assumed in this analysis, but concave upwards, then they may have steep gradients at low frequency and, levelling out to a more or less horizontal asymptote, still allow the forms to be viable at high frequency. The frequency dependence of batesian mimics is of this form, and hence situations with two mimetic forms like that in fig. 1(c) are conceivable (as well as those dealt with as the special case) where all forms are viable at all frequencies, but the equilibrium is nonetheless oscillatory. If the frequency dependence is convex upwards, then the restrictions on lethality are qualitatively the same as in the linear cases, but obviously more extreme.

\section{Special CaSe-The haWk-Dove game}

As an example of the application of the above system to animal conflict, take the hawk-dove game (Maynard Smith, 1978), in which two behavioural phenotypes, fighting "hawks" (H), and "doves" (D) who do not escalate conflicts, meet in pairs to decide possession of some resource, such as food or mates. The fitnesses of the forms are

$$
\begin{aligned}
& W_{\mathrm{H}}=\mathrm{C}+P_{\mathrm{H}} E_{\mathrm{HH}}+P_{\mathrm{D}} E_{\mathrm{HD}} \\
& W_{\mathrm{D}}=\mathrm{C}+P_{\mathrm{H}} E_{\mathrm{DH}}+P_{\mathrm{D}} E_{\mathrm{DD}}
\end{aligned}
$$


where $\mathrm{C}$ is a constant, the $P$ are the phenotype frequencies, $E_{\mathrm{HD}}$ is the mean increment (or decrement) in fitness for a hawk in a conflict with a dove, $E_{\mathrm{DH}}$ the analogous quantity for a dove in a conflict with a hawk, and similarly for $E_{\mathrm{DD}}$ and $E_{\mathrm{HH}}$ (the method for working out the $E$ is described by Maynard Smith (1978)). As the $W$ are obviously linear functions of the phenotype frequencies, all the conclusions of the general case (fig. 1(a)) will apply: in particular $W_{\mathrm{H}}=W_{\mathrm{D}}$ is an equilibrium whatever the type of inheritance, it is not unstable, and it cannot oscillate unless either hawks or doves are totally inviable (or sterile) at high frequencies, which is biologically unlikely. It is a simple matter to show that the equations used here for the general case (equation 16 et seq.) apply to the hawk-dove game if we call hawks form " 0 ", and doves form " 1 ", in which case

$$
\begin{aligned}
s_{0} & =\left(E_{\mathrm{HD}}-E_{\mathrm{HH}}\right) /\left(\mathrm{C}+E_{\mathrm{HD}}\right) \\
s_{1} & =\left(E_{\mathrm{DH}}-E_{\mathrm{DD}}\right) /\left(\mathrm{C}+E_{\mathrm{HD}}\right) \\
t & =\left(E_{\mathrm{HD}}-E_{\mathrm{DH}}\right) /\left(\mathrm{C}+E_{\mathrm{HD}}\right) .
\end{aligned}
$$

The values usually assigned for numerical demonstration $\left(E_{\mathrm{HH}}=-5\right.$, $E_{\mathrm{HD}}=+10, E_{\mathrm{DH}}=0, E_{\mathrm{DD}}=+2$ ) give a low negative value to $s_{1}$, that is to say that the fitness of doves increases with increasing frequency. Provided the lines depicting $W_{\mathrm{D}}$ and $W_{\mathrm{H}}$ still cross as in fig. $1 \mathrm{a}\left(i . e\right.$. , if $\left.\left|s_{1}\right|<|t|\right)$, there is still an equilibrium at $\hat{P}_{\mathrm{H}} / \hat{P}_{\mathrm{D}}=\left(E_{\mathrm{DD}}-E_{\mathrm{HD}}\right) /\left(E_{\mathrm{HH}}-E_{\mathrm{DH}}\right)$ (the solutions to $(6)$ or (18)), and everything in the general case applies. The conditions for oscillation are stringent, as can be seen from the curves for $s_{1}=-0.25$ in figs. 3-5. The fitness of a dove in a dove/hawk meeting must be considerably lower than that of the hawk (very large value of $t$ ), and as the value of $s_{0}$ is always greater than 1 in all three cases, hawks must always do each other so much damage as to be inviable at high frequencies for the equilibrium to oscillate. Doves cannot be inviable at any frequency.

In this case, oscillations will occur only in exceptionally heavily weaponed and aggressive animals which tend to fight to the death, and as with batesian mimicry, will be even less likely if this behaviour is confined to males. In general, the inference that there is a stable equilibrium in an animal conflict game, drawn from examining the invasability of pure populations is correct if

(i) there are two alleles with complete dominance (or haploid inheritance)

(ii) frequency dependence is linear

(iii) neither form is inviable at high frequency.

\section{Conclusions}

In a system with two forms whose fitnesses are negatively frequency dependent, examining the equilibrium behaviour of the system simply by seeing whether both forms can invade a pure population of the other form, of necessity does not provide a complete description. However, the assumption that if invasion by both forms can occur, then there is a stable, non-trivial equilibrium somewhere in the middle, is true under a wide variety of realistic conditions, for a single, two-allele locus. 
Although it is possible to conceive of "ping pong" systems in which the end points are both unstable and there is no non-trivial equilibrium, or in which the single, central equilibrium is also unstable, so that the population is in perpetual, restless motion, as far as I know no biological plausible example has been proposed. It is not however quite so implausible that the central equilibrium shall be oscillatory.

If the functions of frequency dependence are linear, or at least monotonic, then there is a non-trivial equilibrium which is not unstable at the unique point of equal phenotype fitness. But such an equilibrium may be such that displacements produce damped, or even increasing oscillations of frequency around the equilibrium. For instance in the linear case, if $s_{1}=0$, $s_{0}=4, t=0.65$, by the invasion criteria the equilibrium is stable, and so it will be if form " 0 " is recessive; if form " 0 " is dominant, or the system is haploid (Y-linked) then there is a damped oscillation. Although damped oscillations will make rather little difference to the long-term behaviour of the system, undamped ones might have a considerable effect by producing complex limit-cycles of phenotype frequencies. But even damped oscillations are excluded under two rather common circumstances: oscillation is not possible if (i) frequency-dependence of the phenotypes is linear or convex upwards; and (ii) no phenotype can be lethal even at high frequency. The conditions for undamped oscillations are more stringent (they can be found by solving ( 21 ) with -2 in place of -1 ), and if damped oscillations are not permitted, undamped ones are clearly ruled out as well. The condition for an unstable equilibrium (one or both forms with positive frequency dependence, no invasion possible) is obvious.

If frequency dependence is curvilinear and concave upwards, then oscillation is possible without any phenotype being lethal at any frequency. Analysis of the special case of a batesian mimic and a non-mimic shows that the strengths of selection required are very great, probably much greater than occur naturally, and that oscillations are excluded, in the special case only, if mimicry is autosomal and sex-limited. Biologists have of course been surprised in the past by unexpectedly large coefficients of selection!

If the genetic architecture of the trait were not of the simplest type then many of the above conclusions might be vitiated. For example, heterozygotes of intermediate phenotype, or with some physiological disadvantage might lead to systems with three equilibria, and heterozygous advantage by increasing the "stability" might permit oscillations with weaker coefficients of frequency dependence.

Acknowledgements.-I am most grateful to Dr Brian Charlesworth for his helpful suggestions in a draft of this paper.

\section{REFERENCES}

CHARLESWORTH, B., AND CHARLESWORTH, D. 1976. Theoretical genetics of batesian mimicry. I. Single locus models. J. theor. Biol., 55, 283-303.

CHARLESWORTH, B., AND HARTL, D. L. 1978. Population dynamics of the segregation distorter polymorphism of Drosophila melanogaster. Genetics, 89, 171-192.

COOK, L. M. 1971. Coefficients of natural selection. Hutchinson, London.

LEWONTIN, R.C. 1958. A general method for investigating the equilibrium of gene frequency in a population. Genetics, $43,419-434$. 
MAYNARD SMITH, I. 1978. The evolution of behaviour. In Evolution, a Scientific American Book. San Francisco, Freeman.

MAYNARD SMITH, J., AND PRICE, G. R. 1973. The logic of animal conflict. Nature, 246, 15-18.

SLATKIN, M. 1979. The evolutionary response to frequency- and density-dependent interactions. Amer. Nat, 114, 384-398.

TURNER, J. R. G. 1978. Why male butterflies are non-mimetic: natural selection, sexual selection, group selection, modification and sieving. Biol. J. Linn. Soc., 10, 385-432.

TURNER, J. R.G. 1979. Oscillation of gene frequencies in batesian mimics: a correction. Biol. J. Linn. Soc., 11, 397-398. 